\title{
Humanização no atendimento odontológico
}

\author{
Humanization in Dental Care
}

\author{
Diego Teodoro Venancio Lopes ${ }^{*}$, Samantha Peixoto Pereira ${ }^{\circ}$, Tacianne Hott de Souza ${ }^{\circ}$, Tatiana \\ Vasques Camelo dos Santos ${ }^{\circ}$
}

\author{
Centro Universitário Unifacig, Odontologia, Manhuaçu, Minas Gerais, Brasil. *Autor para correspondência. E-mail: \\ diegodih2017@outlook.com
}

\begin{abstract}
Resumo: Introdução: As práticas Odontológicas assumem um papel importante no que diz respeito aos procedimentos cotidianos que os Cirurgiões Dentistas realizam em cada paciente, e ainda o papel fundamental que assumem diante do trabalho humanizado que cada um desempenha, perante cada atendimento realizado acerca dos conhecimentos adquiridos durante sua formação acadêmica. O objetivo do presente estudo busca investigar os conhecimentos que fundamentam as práticas de humanização nos atendimentos odontológicos. Material e métodos: O presente estudo teve abordagem quanti-qualitativa, buscando explorar as práticas humanizadas utilizadas nos atendimentos odontológicos. A coleta de dados se deu por meio de um questionário com questões em padrão aberto e fechado para os Cirurgiões-Dentistas atuantes, no intuito de identificar como suas práticas nos atendimentos odontológicos são humanizadas. Resultados e discussão: Entendimento sobre a Política Nacional de Humanização 84,6\% dos participantes disseram que sabem, sendo que $62 \%$ destes obtiveram tais conhecimentos sobre a Política Nacional de Humanização (PNH) ainda durante o período de formação acadêmica. Quando questionados sobre o entendimento em relação ao que é humanização em saúde, 8,69\% dos participantes associaram a humanização a questões mais amplas, como o Sistema Único de Saúde (SUS), 30,43\% associaram essa prática com considerar o paciente por completo, único e de forma individualizada e 8,69\% fizeram relação com empatia. Em relação ao tempo médio de consulta $76,9 \%$ realizam as suas consultas em um tempo médio de 20 a 40 minutos e 23,1\% realizam suas consultas com tempo acima de 40 minutos, como está descrito no gráfico 4 abaixo. Considerações finais: Contudo é de extrema importância que seja evidenciada a humanização no decurso dos atendimentos odontológicos pelos Cirurgiões Dentistas, de forma que seja aplicada de acordo com as práticas cotidianas em cada paciente.
\end{abstract}

Palavras-chave: empatia, humanização da assistência, odontologia.

\begin{abstract}
Introduction: Dental practices play an important role with regard to the daily procedures that Dental Surgeons perform on each patient, and also the fundamental role they play in the humanized work that each one performs, in the face of each service performed on knowledge acquired during their academic training. The objective this study seeks to investigate the knowledge that underlies humanization practices in dental care. Material and methods: This study had a quantitative-qualitative approach, seeking to explore the humanized practices used in dental care. Data collection was carried out through a questionnaire with open and closed standard questions for practicing dentists, in order to identify how their practices in dental care are humanized. Results and discussion: Understanding of the National Humanization Policy $84.6 \%$ of the participants said they know, and $62 \%$ of these obtained such knowledge about the National Humanization Policy (PNH) during their academic training period. When asked about their understanding of what humanization in health is, $8.69 \%$ of the participants associated humanization with broader issues, such as the Unified Health System (SUS), 30.43\% associated this practice with considering the patient for complete, unique and individualized and $8.69 \%$ related to empathy. Regarding the average consultation time, $76.9 \%$ perform their consultations in an average time of 20 to 40 minutes and $23.1 \%$ perform their consultations with a time over 40 minutes, as described in graph 4 below. Final considerations: However, it is extremely important that humanization is evidenced in the course of dental care by Dental Surgeons, so that it is applied according to daily practices in each patient.
\end{abstract}

Keywords: empathy, humanization of assistance, dentistry.

\section{Introdução}

A Odontologia nos dias atuais, assume um papel muito mais amplo do que em tempos passados, talvez por isso, em teoria, tem-se cobrado dos alunos do curso de odontologia um olhar mais amplo, holístico, no qual 
se considera o paciente como um todo. Entretanto existem inúmeros entraves para o pleno desenvolvimento e formação de profissionais com este enfoque, tais como, na fase de acadêmicos, serem treinados para lidar com ossos, peças cadavéricas e manequins, onde sempre a "boca" está desarticulada do indivíduo. Esses fatores contribuem negativamente para que a sensibilidade na relação profissional-paciente, ainda em formação, seja aguçada desde cedo. Ademais, no documento oficial da Política Nacional de Saúde Bucal (Pucca et al., 2006), afirma-se que "os elementos estruturais ou ideológicos da prática odontológica eram o biologismo, ou seja, excluía a causa social, mecanicismo, centralização dos recursos, tecnificação do ato, ênfase na prática curativa e exclusão das práticas alternativas".

Aliado a tais questões, o impacto das novas tecnologias, que invadiram inúmeros ambientes trabalhistas trazendo a intensificação das características tecnicistas do homem por todo o mundo, proporcionou formas mais técnicas de atendimento e acolhimento dos pacientes pelos profissionais de saúde, reforçando ainda mais a desvalorização do atendimento humanizado no âmbito da saúde pública brasileira. Essa problemática também é vista nos ESFs por todo o Brasil, deixando de lado as necessidades concretas dos usuários dos sistemas públicos de saúde. Frente a isso, a Organização Mundial da Saúde (OMS) sistematizou recomendações curriculares para uma "educação baseada na comunidade" na expectativa de contornar essa questão (Genebra, 2014).

Para Lima (2010) a evolução tecnológica é benéfica, pois segundo ele "o homem já se difere da mais laboriosa abelha e de sua obra perfeita pelo fato de antever o produto de seu trabalho". Assim, tais fatores podem ser percebidos como um ponto desafiador para o alcance de um atendimento holístico e humanizado. Essa questão tem sido tão discutida e vista como realmente importante que o Ministério da Saúde estabeleceu a humanização dos serviços de saúde como um de seus programas prioritários desde 2004. O Programa tem como objetivo contornar essa realidade de atendimentos mais tecnicistas e menos humanizados que tange a área da saúde no Brasil.

De acordo com Waldow (2010) o cuidar é uma atitude ética, que é ensinada durante o processo de formação, permitindo a relação na qual todos os sujeitos possam perceber e reconhecer os direitos uns dos outros. Assim, de forma análoga a esse autor, é evidente que para ter o cuidado, é necessário olhar para si e para os demais, na expectativa de que o autoconhecimento auxilie no cuidado de cada ser.

Dessa forma, preocupa-nos o fato que aspectos relacionados ao atendimento humanizado na odontologia, essenciais ao total sucesso no tratamento, não sejam enfatizados aos cirurgiões dentistas em seu período de formação e muitas vezes também em seus ambientes de trabalho quando já formados, debruçando-se apenas às questões técnicas de seus procedimentos, seguindo os antigos critérios sugeridos pela Política Nacional de Saúde bucal do Brasil.

Diante do exposto, será objetivo deste estudo: investigar os conhecimentos que fundamentam as práticas de humanização nos atendimentos odontológicos.

\section{Materiais e métodos}

O presente estudo teve abordagem quanti-qualitativa, buscando explorar as práticas humanizadas utilizadas nos atendimentos odontológicos. A coleta de dados se deu por meio de um questionário com questões em padrão aberto e fechado para os Cirurgiões-Dentistas atuantes, no intuito de identificar os conhecimentos que fundamentam as práticas humanizadas durante seus atendimentos odontológicos.

O questionário divulgado tinha um total de 22 questões, sendo 14 no formato fechado e as demais perguntas eram abertas. As questões abordaram os seguintes temas: perfil profissional do participante, área de atuação, ano de formação, gênero, raça, idade, ano de conclusão do curso de odontologia, se o participante tinha ou não curso de Pós Graduação, bem como conhecimentos sobre a Política Nacional de Humanização e onde estes foram obtidos (se durante o período de formação acadêmica ou extra curricular). Buscou-se saber do entendimento dos participantes a respeito da humanização em saúde durante seus atendimentos odontológicos e como estes estimulam práticas humanizadas durante seus atendimentos junto aos pacientes, obtendo informações sobre o tempo médio de consulta, investimento em diálogo durante as consultas odontológicas, anamnese ampla considerando todas as demandas dos pacientes, personalização das prescrições odontológicas de acordo com a realidade de cada paciente ,consideração do âmbito familiar durante uma consulta, foco na promoção e prevenção da saúde bucal dos pacientes, ensinamentos direcionados aos pacientes sobre a forma correta de higienização bucal e desejo em se obter cópia das respostas ofertadas.

O convite para participação na pesquisa ocorreu no mês de abril do ano de 2021, sendo divulgado um texto convite, juntamente com o link de acesso ao formulário da pesquisa através das mídias sociais Facebook, 
Instagram e Whatsapp e limitando-se as respostas ao Estado de Minas Gerais. Todos os participantes consentiram em participar do estudo e expressaram seu consentimento por meio de um formulário e também pelo TCLE-Termo de Consentimento Livre e Esclarecido fornecido pelos pesquisadores que estava anexado ao formulário, também no formato online.

Os dados foram analisados estatisticamente e qualitativamente, sendo agrupados em categorias, demonstrando sua relevância para as práticas que fundamentam a humanização nos atendimentos odontológicos.

\section{Resultados e Discussão}

\section{Entendimento sobre Política Nacional de Humanização}

Participaram deste estudo 13 profissionais, destes 23,1\% atuantes na Estratégia Saúde da Família (ESF) e $76,9 \%$ atuantes na iniciativa privada.

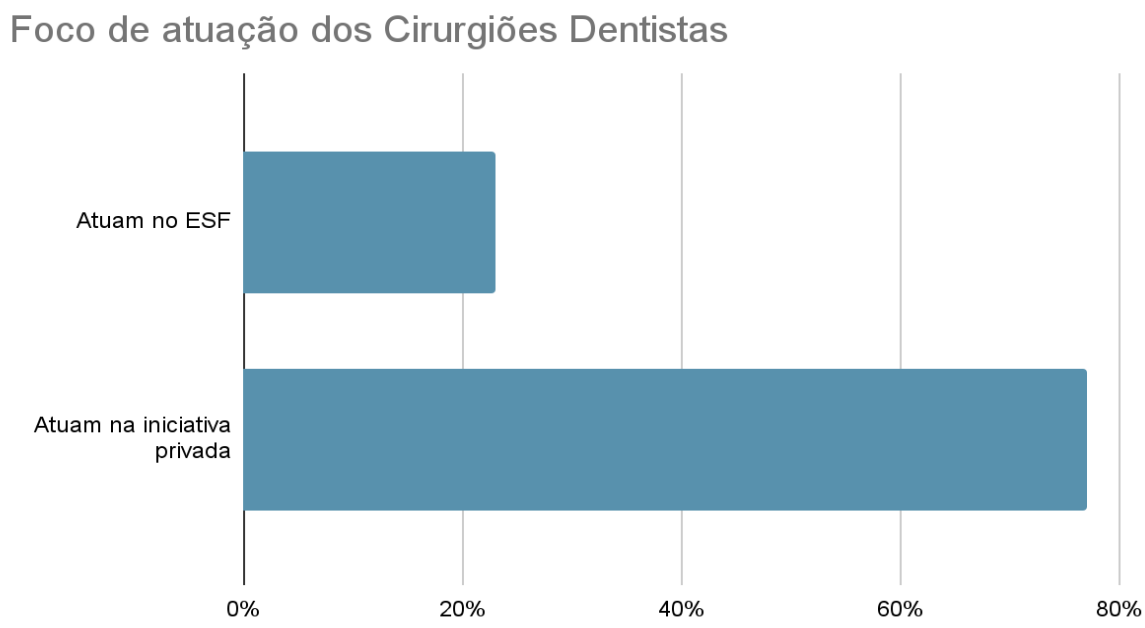

Figura 1. Foco de atuação dos Cirurgiões Dentistas. Fonte: Autores/Dados da pesquisa, 2021.

O gráfico 2 demonstra a resposta dos participantes à pergunta "Você sabe o que é Política Nacional de Humanização?”, na qual 84,6\% dos participantes disseram que sabem, sendo que 62\% destes obtiveram tais conhecimentos sobre a Política Nacional de Humanização (PNH) ainda durante o período de formação acadêmica, conforme consta no gráfico 3.

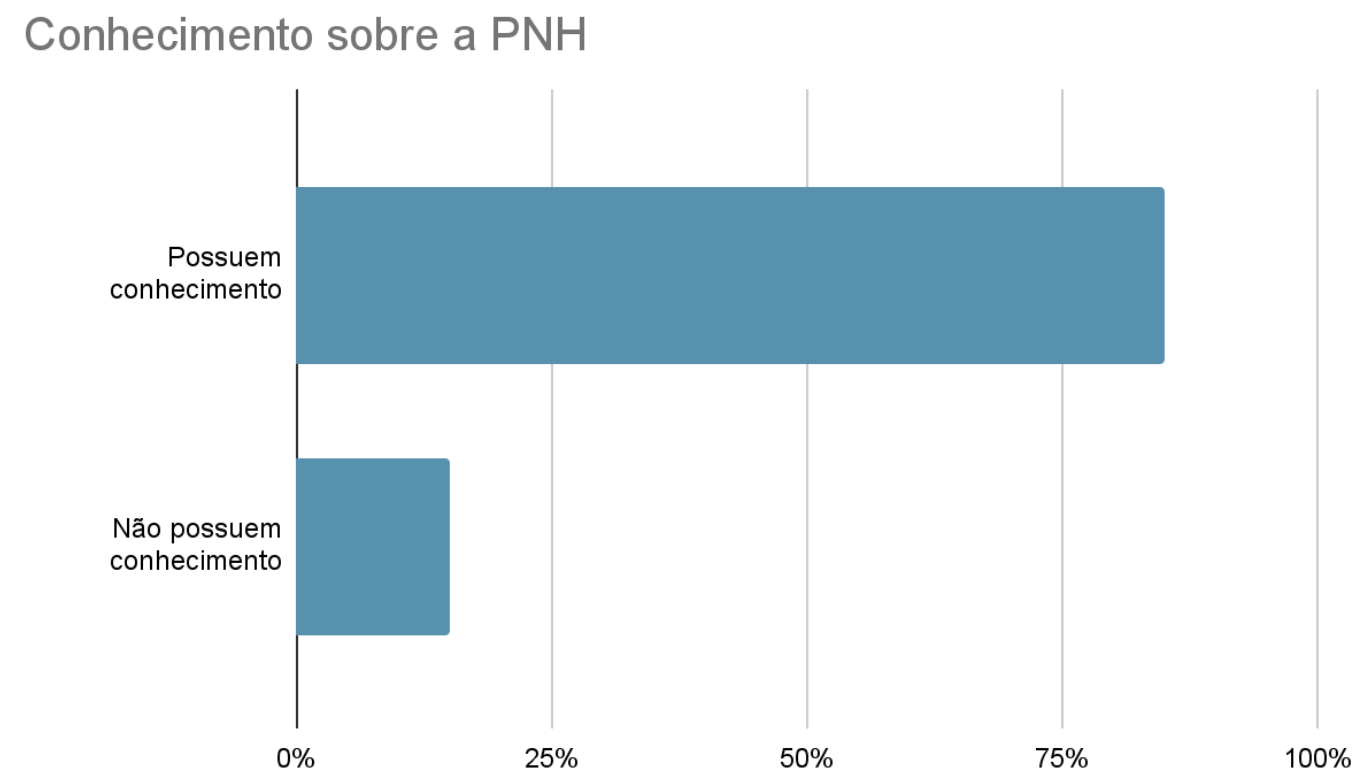

Figura 2. Conhecimento sobre a PNH. Fonte: Autores/Dados da pesquisa, 2021. 
Conhecimentos transmitidos sobre a PNH durante a formação

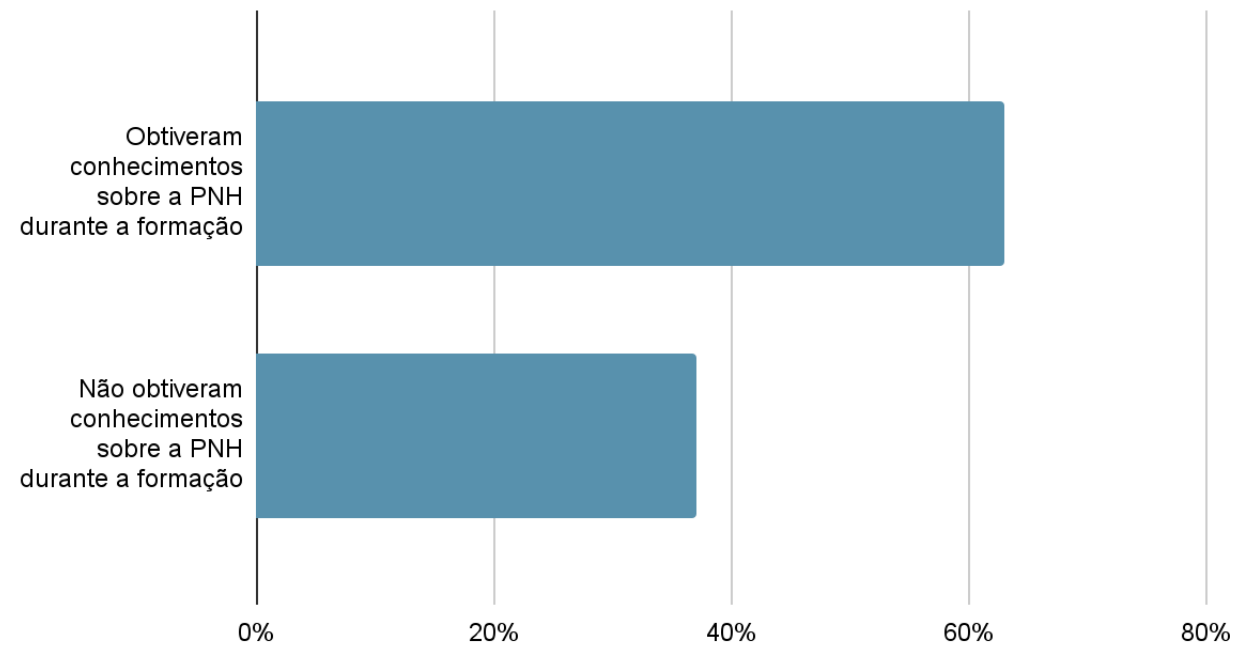

Figura 3. Conhecimentos transmitidos sobre a PNH durante a formação. Fonte: Autores/Dados da pesquisa, 2021.

A Política Nacional de Humanização (PNH) foi elaborada pelo Ministério da Saúde em 2003 com o objetivo de transformar o modelo de atendimento em saúde a partir de técnicas transversais incluídas em todas as redes do Sistema Único de Saúde (SUS), na tentativa de superar aquele antigo modelo tecnicista que reverbera até hoje na prática clínica de inúmeros profissionais da saúde em seu âmbito de trabalho (Santos Filho et al., 2009; Pasche et al., 2011). Dessa forma, é evidente que a maior parcela dos participantes conseguiu absorver conteúdos sobre a PNH durante o período de formação, o que provavelmente pôde contribuir positivamente para a superação do antigo modelo de atendimento evidenciado por (Santos Filho et al., 2011).

Quando questionados sobre o entendimento em relação ao que é humanização em saúde, 8,69\% dos participantes associaram a humanização a questões mais amplas, como o Sistema Único de Saúde (SUS), 30,43\% associaram essa prática com considerar o paciente por completo, único e de forma individualizada e 8,69\% fizeram relação com empatia. Tendo em vista que apenas uma pequena parcela dos participantes relacionou humanização com empatia, é notório que esta é uma questão a ser analisada. Para Leal, a empatia é um elemento central nas relações entre profissionais da saúde e seus pacientes, gerando uma contribuição para melhor compreensão dos inúmeros aspectos das interações sociais em saúde. Assim, a falta da associação da humanização com a prática da empatia, pode gerar uma lacuna entre o profissional e o paciente durante uma interação, provocando possíveis reflexos negativos durante o tratamento.

Para (Oriá et al., 2004), a atenção a todas as necessidades sejam elas, biológicas, psicológicas, sociais, espirituais e emocionais do usuário é definida como cuidado holístico, e considerada como fundamental para um processo de comunicação eficiente. Assim, se relacionada com o atendimento humanizado, traz inúmeros benefícios. Portanto torna-se evidente que a falta de conhecimento sobre a PNH e suas diretrizes impactam de forma negativa as ações para com o paciente.

\section{Práticas Humanizadas em Odontologia}

Constatou-se que $84,6 \%$ dos entrevistados nesta pesquisa realizaram questionamentos durante a anamnese voltados a condições financeiras, âmbito familiar e qualidade de vida do paciente, e 100\% alegaram considerar as relações de âmbito familiar e social de seus pacientes. De acordo com os dados obtidos, nota-se uma porcentagem positiva voltada às práticas humanizadas em Odontologia, mostrando um cenário de atendimento odontológico compatível com as diretrizes da Política Nacional de Humanização (PNH).

Em paralelo a esses dados obtidos, podemos mencionar o estudo realizado por Araújo (2008), já que para ele o profissional deve analisar o indivíduo de maneira que seja um "ser" e não somente cuidar da sintomatologia, considerando todas as suas relações. Neste sentido, as ações de acolhimento na odontologia, são consideradas fundamentais para uma melhor qualidade de assistência que será prestada, sendo uma característica notória de um atendimento humanizado, pois quando se acolhe um paciente, se inicia uma relação, cria-se um vínculo entre o acolhido (paciente) e toda a equipe que prestará os serviços. 100\% dos 
participantes alegaram proporcionar ao paciente segurança, relatando todos os procedimentos e suas etapas durante a realização dos mesmos. De acordo com Moimaz et al. (2015) o profissional que não possui o hábito de transmitir informações ao paciente sobre o tratamento que será prestado gera índices variados de insatisfação. Os autores também consideraram que a ansiedade dos pacientes durante qualquer procedimento odontológico tende a diminuir consideravelmente quando se é colocado em prática o modelo de humanização nos serviços de saúde. Portanto, é evidente que as práticas humanizadas nos atendimentos odontológicos são de suma importância para o sucesso no tratamento, sendo fundamental conhecer o indivíduo por completo em sua totalidade.

\section{Tempo médio de consulta odontológica}

Os participantes foram questionados sobre a duração de suas consultas odontológicas, assim foi constatado que $76,9 \%$ realizam as suas consultas em um tempo médio de 20 a 40 minutos e $23,1 \%$ realizam suas consultas com tempo acima de 40 minutos, como está descrito no gráfico 4 abaixo.

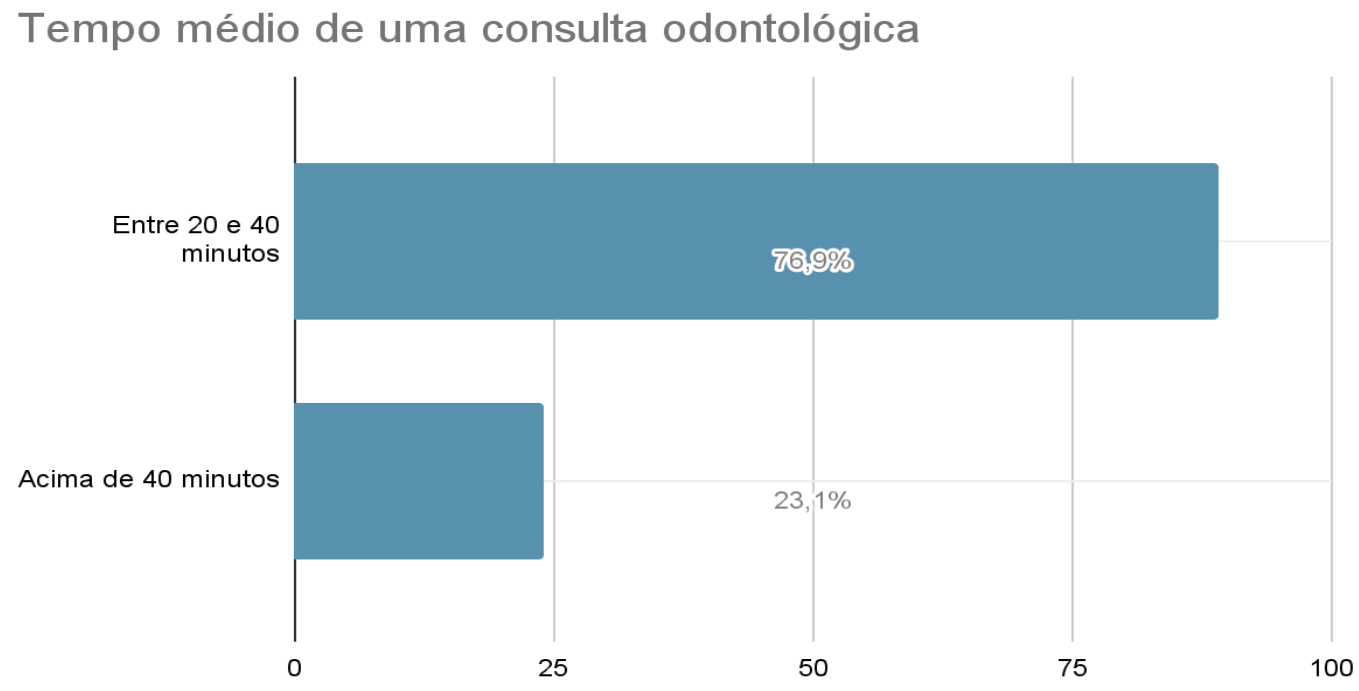

Figura 4. Tempo médio de uma consulta odontológica. Fonte: Autores/Dados da pesquisa, 2021.

De acordo com a American Academy of Pediatric Dentistry (1997; Academia Americana de Odontopediatria) dez aspectos são bem importantes para uma boa qualidade na assistência odontológica, sendo eles:

1. Responsabilidade: O cirurgião Dentista deve ser responsável e ter íntima participação durante a avaliação da assistência prestada.

2. Estabelecimento de aspectos fundamentais da assistência que farão parte da avaliação.

3. Identificação de casos de grande impacto na qualidade do cuidar, pacientes de alto risco, alta atividade cárie dentária e problemas periodontais.

4. Utilização de indicadores de qualidade de assistência no intuito de ajudar na identificação de problemas e formas de melhoria no cuidado prestado.

5. Estabelecimento de metas para cada forma de indicador que será desenvolvido.

6. Realização de uma coleta coesa e precisa e organização de todos os dados.

7. Avaliação da assistência a partir de comparações de antigos resultados com as metas previamente estabelecidas.

8. Conhecimento da assistência adequada, possíveis novas estratégias para superação de problemas existentes.

9. Avaliação corriqueira das estratégias traçadas.

10. Comunicação das informações.

Todos esses aspectos listados, que são de fundamental importância para uma boa qualidade no cuidado odontológico prestado, evidenciam a necessidade de um tempo coerente para que um amplo cuidado seja realizado. Para Benazzi et al. (2010) uma das características percebidas pelos usuários em atendimento são: cordialidade no atendimento, tempo de espera, falta de profissionais e consultas rápidas. 
De acordo com os dados demonstrados no gráfico 4, fica evidente que partindo de uma avaliação temporal e com o auxílio de trabalhos já publicados, constata-se que quanto maior for o tempo de uma consulta odontológica, maior será a coleta de dados para uma ampla anamnese, bem como uma maior relação profissional-paciente será estabelecida. A qualidade de serviços assistenciais está associada à qualidade da relação interpessoal que ocorre entre os pacientes e os profissionais, essa forma de trabalho analisa aspectos psicológicos envolvidos na relação Cirurgião Dentista- Paciente, contribuindo no aprimoramento de uma assistência com maior foco em especificidades não tão evidentes que cada paciente apresenta. Para que os serviços prestados incluam todos os caracteres já mencionados, sendo um dos primordiais,o fator tempo de uma consulta, o profissional necessita possuir responsabilidade profissional, experiência, compromisso com a organização, com os usuários e com as atividades que serão desenvolvidas.

\section{Considerações finais}

Evidenciou-se que a humanização nos atendimentos odontológicos é de extrema importância para sucesso no tratamento. Humanizar não é um conceito restrito unicamente a uma ação, mas sim, se trata de inúmeras medidas fundamentadas em conhecimentos que podem proporcionar ao profissional sensibilidade para conseguir identificar e lidar com as especificidades de cada paciente que a ele for destinado.

Dessa forma, foi notado que durante o período de formação acadêmica a temática humanização é presente, entretanto observa-se uma diferença na forma e na intensidade de transmissão desses conhecimentos. Isso faz com que o termo humanização em saúde se torne difundido entre os profissionais de uma forma ligeiramente desigual e que quando identificado por eles sempre está relacionado a uma única medida ou tomada de decisão, mas quase nunca visto e entendido como um conceito bem amplo e com inúmeras relações com a saúde, propiciando uma complexidade em sua prática no cotidiano clínico dos profissionais. De uma forma ampla, os dados coletados conseguem evidenciar um cenário de atendimentos odontológicos consideravelmente de acordo com o que se deve esperar de um atendimento humanizado, mas é válido ressaltar, que por se tratar de uma pesquisa com um público participativo reduzido e restrito apenas ao Estado de Minas Gerais, tais resultados não devem ser considerados como base estatística para outros campos de pesquisa nos quais podem ser encontrados um público participativo maior. Assim, é fundamental que esse trabalho sirva como fonte fomentadora da busca por mais conhecimentos sobre essa abordagem que é de suma importância para o âmbito da saúde no Brasil, incentivando a publicação de outros trabalhos com relação direta a esse. Somente assim, fontes de conhecimentos teóricos sobre o tema estarão presentes de forma ampla para o acesso de inúmeros profissionais de saúde, tanto os em formação quanto os em atuação, fazendo essa abordagem ser popularizada e vista como realmente necessária e principalmente colocada em prática.

\section{Referências}

Araújo, M. F. S. 2008. Reflexões sobre a prática em Odontologia. São Paulo, SP: Artes Médicas.

Benazzi, L. E. B., Figueiredo, A. C. L., \& Bassani, D. G. 2010. Avaliação do usuário sobre o atendimento oftalmológico oferecido pelo SUS em um centro urbano no sul do Brasil. Ciência \& Saúde Coletiva, 15, 861-868.

Genebra, T., Santos, R. R., Francisco, R., Pinto-Marijuan, M., Brossa, R., Serra, A. T., ... \& Zarrouk, O. 2014. Proanthocyanidin accumulation and biosynthesis are modulated by the irrigation regime in Tempranillo seeds. International Journal of Molecular Sciences, 15(7), 11862-11877.

Lima, E. D. N. D. A., \& Souza, E. C. F. D. 2010. Percepção sobre ética e humanização na formação odontológica. RGO - Revista Gaúcha de Odontologia, 58(2), 231-238.

Moimaz, S. A. S., de Toledo Rós, D., Bordin, D., Rovida, T. A. S., \& Garbin, C. A. S. (2015). Satisfação e perfil de usuários do serviço odontológico no Sistema Único de Saúde. Revista da Faculdade de Odontologia-UPF, 20(3).

Oriá, M. O. B., Moraes, L. M. P., \& Victor, J. F. 2004. A comunicação como instrumento do enfermeiro para o cuidado emocional do cliente hospitalizado. Revista Eletrônica de Enfermagem, 6(2), 292-295.

Pasche, D.F., Passos, E., \& Hennington, E.A. 2011. Cinco anos da política nacional de humanização: trajetória de uma política pública. Ciência \& Saúde Coletiva, 16(11), 4541-4548.

Pucca Junior, G.A. (2006). A Política nacional de saúde bucal como demanda social. Ciência \& Saúde Coletiva, 11, 243-246. 
Santos Filho, S. B., Barros, M. E. B. D., \& Gomes, R. D. S. 2009. A Política Nacional de Humanização como política que se faz no processo de trabalho em saúde. Interface-Comunicação, Saúde, Educação, 13(1), 603-613.

Waldow, V. R. 2010. Cuidar: expressão humanizadora da enfermagem ( $3^{\mathrm{a}}$ ed.). Petrópolis, RJ: Vozes.

\section{Minicurrículo}

Diego Teodoro Venâncio Lopes. Acadêmico do $4^{\circ}$ período do curso de Odontologia do Centro Universitário Unifacig,Manhuaçu.MG,Brasil.

Samantha Peixoto Pereira. Cirurgiã Dentista. Doutora em Clínicas Odontológicas, com ênfase em Periodontia pela UNITAU. Mestre em Ciências da Educação - UNINORTE. Especialista em Gestão de PSF Programa da Saúde da Família pela FIJ. Professora do curso de Graduação em Odontologia do Centro Universitário Unifacig.

Tacianne Hott de Souza. Acadêmica do $4^{\circ}$ período do curso de Odontologia do Centro Universitário Unifacig, Manhuaçu, MG, Brasil.

Tatiana Vasques Camelo dos Santos. Enfermeira. Mestre em Enfermagem pela Universidade Federal do Rio de Janeiro - UNIRIO. Doutora em Enfermagem na linha de Planejamento e Gestão dos Serviços de Saúde pela Universidade Federal de Minas Gerais - UFMG. Professora dos cursos de Graduação e Pós Graduação do Centro Universitário Unifacig.

Como citar: Lopes, D.T.V., Pereira, S.P., Souza, T.H., \& Santos, T.V.C. 2021. Humanização no atendimento odontológico. Pubsaúde, 7, a245. DOI: https://dx.doi.org/10.31533/pubsaude7.a245

Recebido: 25 ago. 2021.

Revisado e aceito: 07 set. 2021.

Conflito de interesse: os autores declaram, em relação aos produtos e companhias descritos nesse artigo, não ter interesses associativos, comerciais, de propriedade ou financeiros que representem conflito de interesse.

Licenciamento: Este artigo é publicado na modalidade Acesso Aberto sob a licença Creative Commons Atribuição 4.0 (CC-BY 4.0). 